\title{
MIT DER SÜDOSTBAHN NACH KÄRNTEN UND SALZBURG
}

\author{
Herbstreise $195 \mathrm{I}$ der Geographisch-Ethnographischen Gesellschaft Zïrich
}

Jost HösLI

\begin{abstract}
Vom 10. bis 14. Oktober 1951 flitzte eine sonderbare Zugskomposition über die elektrifizierten Schienenwege des westlichen Oesterreichs. Drei moderne Personenwagen um eine Lokomotive gruppiert, eine Südostbahn im Lande der Nord-, Ost-, Süd- und Westbahnen! Die Anschrift erregte nicht weniger Staunen als die ungewohnte Formation. Darob wurden die gastfreundlichen Nachbarn so wenig weise wie die Zürcher, die sich frugen, was wohl die SOB in der Limmatstadt zu tun habe. Das Rätselraten galt den bereits erwähnten Requisiten einer fünftägigen Exkursion nach Oesterreich, die von der Südostbahn zusammen mit der Geographisch-Ethnographischen Gesellschaft Zürich veranstaltet wurde. Das Programm verhieß viel, die Fahrt bot mehr. Der schmucke Sonderzug sorgte für Publizität und Popularität. Er erleichterte unliebsame $Z$ ustandsveränderungen, welche immer müde und auch nervös machen: Paßkontrolle, Zoll! Und er ermöglichte eine unterhaltsame Art des Reisens, die selbst die Verwöhnten unter den Teilnehmern begeisterte. Dank gebührt daher den Menschen, die trefflich verstanden, vielfältige Mittel am rechten Ort und zur rechten Zeit einzusetzen. Ihre freudige Bereitschaft war mehr als bloße Pflichterfüllung. Die Anerkennung gilt einmal der Direktion der SOB, welche die Hauptlast der Organisation übernahm: Dir. Dr. ZuBER und seinem Famulus Sekretär E. MúlLER, den sympathischen Angestellten wie auch dem Vertreter der österreichischen Verkehrszentrale und den fremden Bahnbeamten, die "Lotsendienste “ leisteten. Dem „Administrator “ der Gesellschaft, Dr. E. Schwabe (Zürich), standen vom ersten Tage an die Salzburger Wissenschafter Prof. Dr. K. Willvonseder und Dr. E. LendL zur Seite. Ihre vorsorglichen Bemühungen und lehrreichen Erklärungen vertieften das Erlebnis von Land und Leuten und trugen so viel zum herrlichen Gelingen der Studienfahrt bei.
\end{abstract}

In kurzer Zeit ließ der Zug die Grenze bei Buchs hinter sich. Die steile Arlbergrampe führte ihn aus den Nebeln der Niederungen ins volle Herbstlicht der Bergwelt. Derselbe Glanz lag auf den Tallandschaften jenseits der Wasserscheide. Tiefblauer Himmel überdachte die ungewohnt hellen Kalkberge, an deren Flanken Tannenwälder bis an die Schutthalden und schroffen Wände klimmen. Ihr dunkles Grün umrandet das farben- und formenreiche Mosaik der Talgründe. Von Mädchen und Frauen gehütet, weidete auf den Wiesen braunes und rotfleckiges Vieh. An Heinzen trocknete das letzte Emd. Wie das Rheintal, so ist auch das Inntal eine bekannte Föhnrinne. Männer schnitten den Mais und bündelten Stengel und Blätter zı großen Puppen. An Histen hingen die rotgelben Kolben, oft ganze Häuserfassaden kleidend. Von den Schuttkegeln der Sonnenhänge grüßen die weißgetünchten Kirchen der Dörfer. Die Türme tragen entweder gotische Spitzen oder barocke $Z$ wiebein. Bei $Z$ irl weitet sich der 'Taltrog zum breitsohligen und terrassenreichen Unterinntal. Nun reihen sich kulturreiche Städte am breiten Inn: Innsbruck, die alte Hauptstadt vom Tirol, am Schnittpunkt der Arlberg-, Brenner: und Mittenwaldroute gelegen. Hall, berühmt durch den bis in vorrömische Zeiten zurückreichenden Salzbergbau, Schwaz, wo einst Silber und Kupfer gefördert wurde, Jenbach, die Pforte zum Achensee und Zillertal, und das direkt am Fluß gelegene reizvolle Rattenberg.

Von Wörgl führen bequeme Talwasserscheiden durch das Brixen, Lenken und Leoganger Tal ins mittlere Pinzgau, über Kitzbühel, St. Johann nach Saalfelden an der Saalach. Isolierte Kalkstöcke wachsen aus der Landschaft: das Kaisergebirge, die Loferer- und Leoganger Steinberge, das Steinerne Meer. Nach Süden vermittelt eine eiszeitlich übertiefte Quertalrinne den Zugang zum Längstal der Salzach, dem Ober- und Unter Pinzgau. In ihr liegt Zell am See. Wiesenreiche Hänge deuten die Schiefer- und Grauwacken der leicht verwitterbaren «Grasberge » an. Behäbige Bauernhäuser überstreuen die von charakteristischen Holzzäunen gemusterte Wiesensohle. 


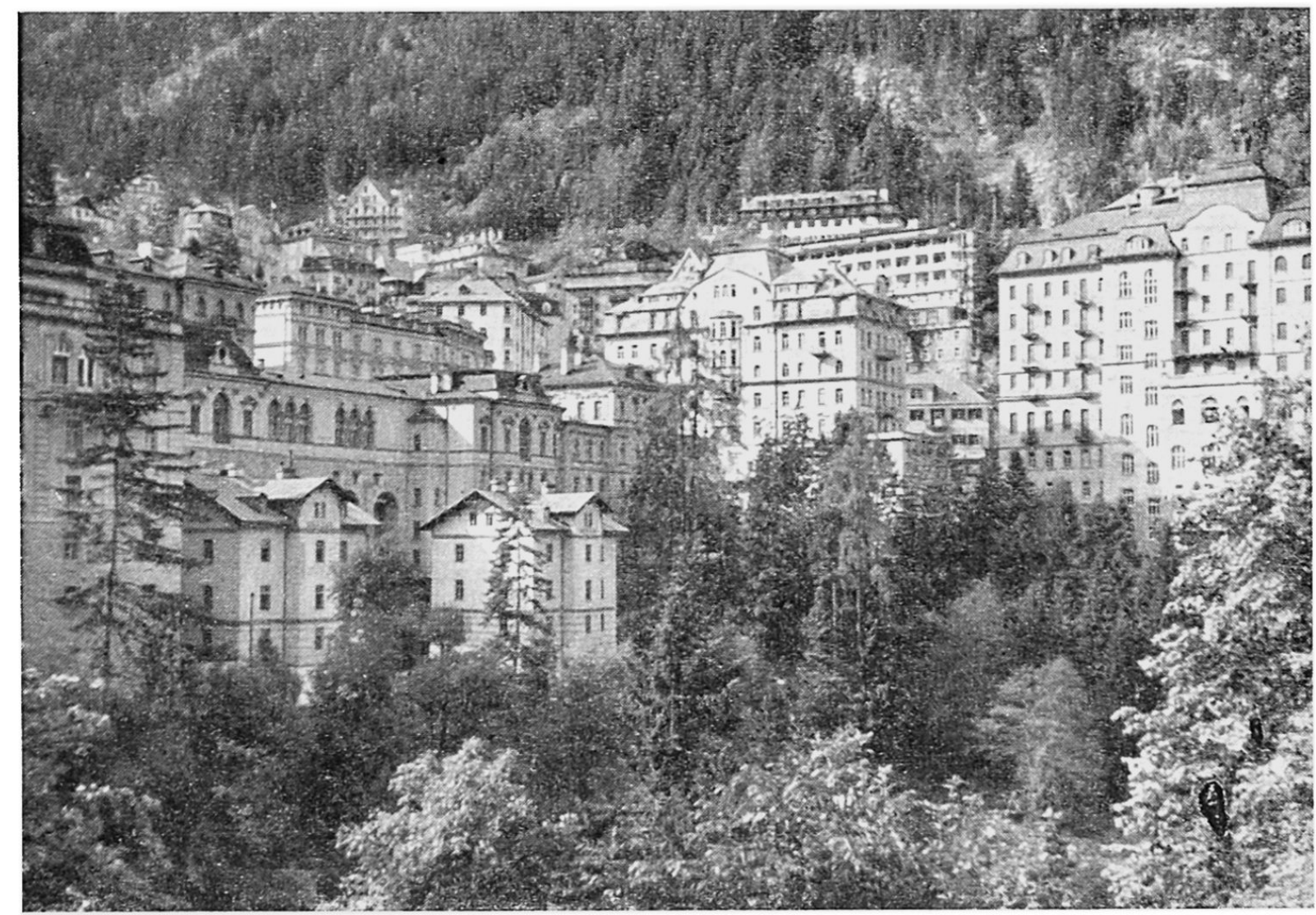

Hotelstadt Bad Gastein. Blick gegen die westliche Talflanke am Stubnerkogel $(2248 \mathrm{~m})$ Photo J. HösLI, 12. 10. 51

Von Bruck an der Großglocknerroute nach dem Verkehrsknoten SchwarzachSt. Veit an der Tauernlinie (Salzburg-Villach) fließt die Salzach durch ein schluchtartiges Tal. Nach der Spitzkehre in Schwarzach rollt der Zug durch das Tal der Gasteiner Ache den aus Granit und kristallinen Schiefern gefügten Tauern entgegen, welche dem Aar- und Gotthardmassiv vergleichbar das Zentralgebirge der Ostalpen bildet. Der 1906 bis 1909 erbaute, 8,5 km lange Tunnel durchsticht auf rund $1200 \mathrm{~m}$ Höhe die östliche Gruppe der Hohen Tauern (Ankogel $3246 \mathrm{~m}$ ). Die Südrampe erinnert an die Lötschbergstrecke Goppenstein-Brig. Dem linken Hang des engen Mölltales entlang erreicht der Schienenstrang endlich bei Spittal den Talgrund, die breite Talsohle der am Ostfuß der Dolomiten entspringenden Drau. Bei Villach tritt der stattliche Fluß in eines der für die Ostalpen typischen Tertiärbecken ein: die Kernlandschaft von Kärnten. Gegen Süden wird das vom einstigen Draugletscher geprägte, seenreiche Becken durch die imposante Grenzmauer der Karawanken abgeschlossen. Bei einbrechender Nacht manöverierte eine Dampflokomotive den Extrazug von Villach nach Velden am Wörthersee, wo die Reisegesellschaft im prächtigen Schloßhotel Unterkunft fand.

Kärnten liegt so südlich wie das Südtirol. Klagenfurt hat ungefähr die geographische Breite von Meran und Bozen. Die jugoslavisch-österreichische Karawankengrenze liegt kaum $100 \mathrm{~km}$ von der Adria (Triest) entfernt. Zu Tausenden trocknen im Herbst die Maiskolben an Scheunen und Wohnhäusern. Kärnten liegt kontinental, Hitze und Kälte wechseln jahreszeitlich miteinander ab. An die Wärme erinnerte allein das mächtige Lob des Sommers und das Rotgold der Maiskolben. Die Kälte aber wurde allen zum tiefgreifenden Erlebnis, denn der zweite Tag demonstrierte wirklichen Winter. Hochnebel verschleierten Himmel und Berge und eine ungewohnte Frische begleitete über Land, nach dem Fakkersee, nach Vil- 
lach und an den Ossiachersee. Die mit einer Luftseilbahn erreichbare, $1500 \mathrm{~m}$ hoch gelegene Kanzelhöhe ist eine berühmte Aussichtswarte. Sie, die «mehr Sonnenscheinstunden und intensivere UV-Strahlung aufweist als Davos oder Arosa », lag im dichten Nebel, aus dem leicht Schnee fiel. Über die am.Nordufer des rund $20 \mathrm{qkm}$ messenden Wörthersees gelegenen Sommerkurorte Velden und Pörtschach war bereits der Winterschlaf gekommen. In den Straßen Klagenfurts hantierten die Kastanienbrater. Wie ihre Ware, so wurden auch die geheizten Räume des im Renaissancestil gehaltenen Landhauses (1547) geschätzt. Der Tag schloß mit einer luftigen Pilgerfahrt über den See nach Maria Wörth.

Die Etappe des dritten 'Tages hieß Salzburg. Die Tauern funktionierten als Klimascheide; was der Süden nicht schenkte, bescherte der Norden. Über dem Gasteiner Tal blaute der Himmel, der Fachmann registrierte Föhn. Im weltberühmten Bad Gastein, einer amphitheatrajisch um einen $65 \mathrm{~m}$ hohen Wasserfall gruppierten Kursiedlung entbot ihr Bürgermeister den Gästen herzlich Willkomm. Das Salzachquertal ab Schwarzach-St. Vcit erschloß wiederum « Neuland ». Auf St. Johann im Pongau folgt Bischofshofen. Hoch über Werfen thront die prächtige Burg, ehemalige Wehrbaute der Erzbischöfe von Salzburg. Das Tal verengt sich, die schroffen Köpfe des Tennengebirges treten dem Fluß, der Schiene und Straße immer näher. Bald hat nur noch die gischtende Salzach Platz. Die Schlucht heißt $\mathrm{Pa} \beta$ Lueg. Auf ihren engen Schlund folgt ganz unerwartet die breite, weite Salzburgerebene. Bald grüßt die Salzstadt Hallein über die Wasser. Und in der Ferne des offenen Alpenvorlandes kündet die weit sichtbare Feste Hohensalzburg die Stadt der Festspiele an.

Eine von den Salzburger Geographen in zuvorkommender Weise organisierte Ausstellung orientierte über die begonnenen Arbeiten zu einem Heimatatlas. Dann bestieg man das „Wasserbähnchen " und damit den imposanten Burghügel $(542 \mathrm{~m})$, dessen Bauten die kirchenreiche Altstadt $(424 \mathrm{~m})$ krönen. Im sicheren Winkel des schwer zugänglichen steilen Schloßfelsens und der in seinem Lee erhalten gebliebenen Mindel-Riß Schotterrippe Mönchsberg gelegen, markiert diese den geologischen Rand der nördlichen Kalkalpen, der vom Hohen Staufen $(1773 \mathrm{~m})$ im Westen über die Inselberge Hohensalzburg und Kapuzinerberg nach dem Nocksteinzug $(1040 \mathrm{~m}) \mathrm{im}$ Osten verläuft. Das Abendlicht vergoldete das prächtige Panorama. Im Norden das mächtige Flachland der schwäbisch-bayrischen Hochebene, im Süden die vielgliedrigen Ostalpen! Salzburg als beider Naht ist Mittlerin, ein edles Portal, an dem Erd- und Menschengeschichte gebaut haben und an dem eine lebendige Gegenwart weiterbaut. Bald lag das fürstbischöfliche Salzburg im Schatten. Aus seinen engen Gassen glitzerten die ersten Lichter durch den über den Dächern schwebenden Rauchschleier. Bei einbrechender Nacht durchschweifte man gruppenweise die Altstadt, um weniges von ihrem köstlichen Inhalt zu erleben.

Der Samstag bot den Höhepunkt der Reise: das liebliche Salzkammergut im Festgewand eines unvergleichlich schönen Herbsttages. Dem morphologisch auffälligen Kalkalpenrand entlang surrten die Cars der Sonne entgegen. Wie Kliffs ragen aus der wiesen- und waldgrünen Flyschlandschaft des Alpenvorlandes die Zeugen der tirolischen Deckenfront. Vom bereits erwähnten Nockstein zieht sich die oberostalpine Überschiebungsstirn durch eine stark gestörte Zone nördlich des Fuschlsees zum Schober $(1329 \mathrm{~m})$ und zur Drachenwand an das Südufer des Mond- und Attersees hin. Dazwischen öffnet sich von SE die von einem Arm des Traungletschers ausgeschürfte Talung des Fuschl- und St. Wolfgangsees. Der inmitten eines wahren Seenkranzes gelegene Schafberg $(1780 \mathrm{~m})$ ist die Rigi des Salzkammergutes. Die Dorfkirche des populären Fremdenortes St. Wolfgang (Weißes Rößl!) birgt den herrlichen Flügelaltar von Michael Pacher. Die trefflichen Begleitworte des Salzburger Geographen Prof. Dr. J. LeCHNeR vertieften die Bewunderung des 1479-81 geschaffenen, die Krönung Marias verherrlichenden Kunstwerkes. Über Bad Ischl und Ebensee wurde zur Mittagszeit Gmunden am Ende des Traunsees erreicht. Einem luftigen Abstecher am Seil auf die latschenreichen Höhen des Feuerkogels $(1600 \mathrm{~m})$ im Höllengebirge folgte die Rückfahrt durch die buntgefärbten Waldungen des Weißbachtales. Volles Mondlicht spielte im Mondsee und beglei- 


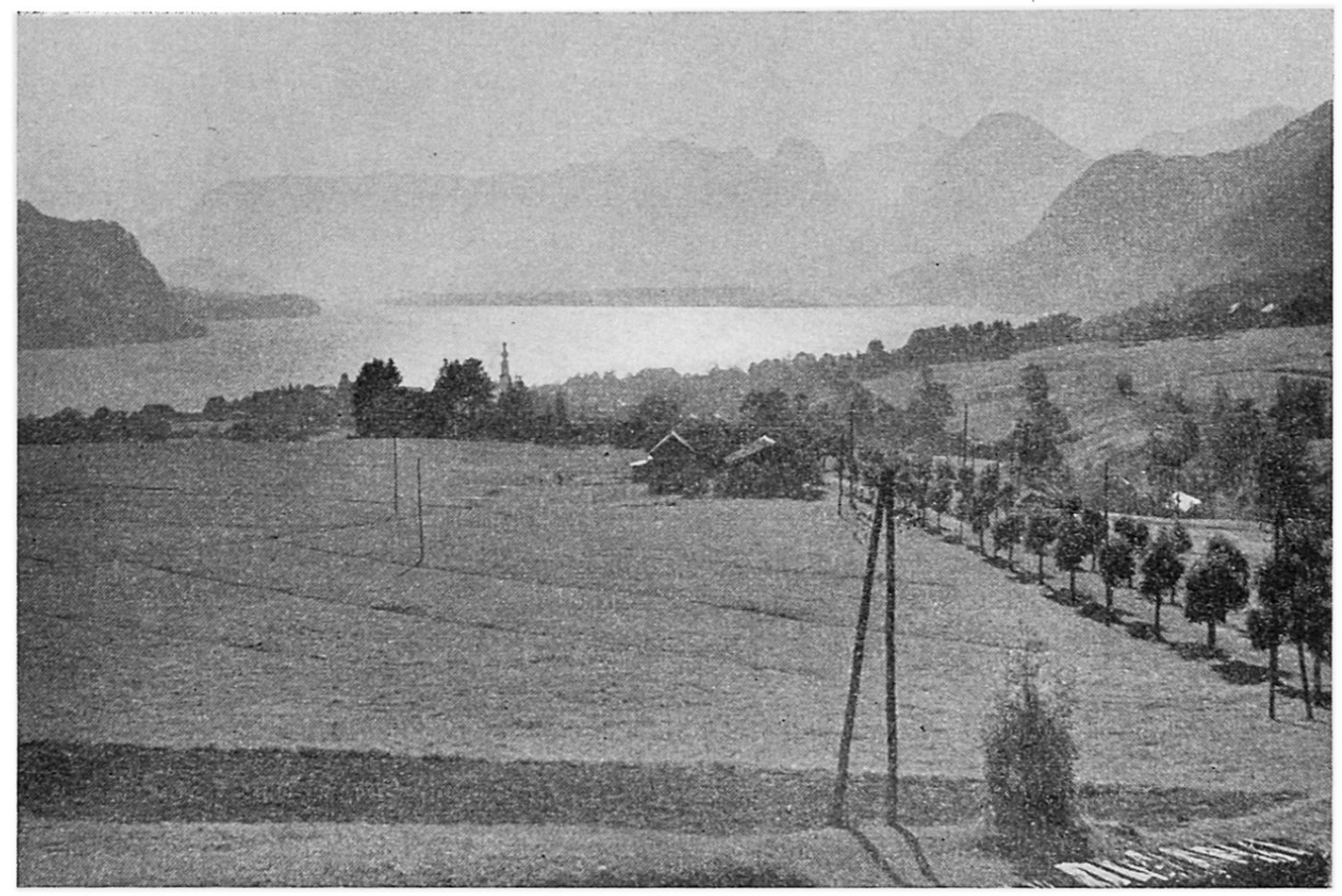

Wolfgangsee im Salzkammergut. Von Westen. Im Mittelgrund St. Gilgen.

Photo E. SchwaBe, 13. 10.51

tete die Heinkehrer durch eine zauberhafte Schattenlandschaft nach der Mozartstadt zurück. Der Abend vereinigte die Salzburger und Schweizer Fachgeographen $\mathrm{zu}$ einer gemütlichen Zusammenkunft, welche mit dem Besuch des Landeshauptmanns Dr. Josef Klaus beehrt wurde.

Der letzte Tag führte am Chiemsee vorbei und dem Alpenrand entang nach dem bayrischen Rosenheim am Inn. Eine mächtige Nebelzunge erfüllte die Talung. Doch brachte die Strompforte Kufstein gleichzeitig mit der österreichischen Erde auch die leuchtende Sonne zurück. Talaufwärts über Wörgl, wo der Kreis der Reise sich schloß, wurde Innsbruck erreicht. Ihm galt der letzte Halt. Autos entführten die hungrige Schar nach der am Gebirgsfuß der Karwendelgruppe gelegenen Aussichts- und Gaststätte Hungerburg. Beim Mittagsmahl wurden die Innsbrucker Professoren Dr. R. v. Klebelsberg und Dr. H. Kinzl begrüßt und mit launigen Worten des Dankes das Ende der gelungenen Herbstfahrt gefeiert. Auf der Rückfahrt in die Stadt ließ Prof. v. Klebelsberg, angesichts der wissenschaftlich bedeutsamen Höttinger Breccie in souveräner Art die geologische Entwicklung der Landschaft erstehen. Für die reiche Kulturschätze bergende Stadt blieb leider wenig Muße. Die unverrückbare Abfahrtszeit beschnitt die Abstecher zum Goldenen Dachl und in die Hofkirche zum Grabmal Kaiser Maximilians I nur zu rasch. Förmlich aus dem Staunen gerissen, hatte man sich wieder dem Räderwerk der Technik einzuordnen. «Partir, c'est mourir un peu!»

\section{DIE SCHWEIZ. GEOMORPHOLOG ISCHE GESELLSCHAFT I M AARGAUISCHEN REUSSTAL}

Unter der Leitung von Dr. H. JAEckl, Zürich, fand am 1. und 2. September 1951 die traditionelle Sommerexkursion statt, deren Zweck das Studium der Talgeschichte und der Abtragungs- und Aufschüttungsformen zwischen Bremgarten 